\title{
ON A THEOREM OF HIGHER RECIPROCITY*
}

\section{BY LEONARD CARLITZ}

1. Introduction. Let $\mathfrak{D}$ denote the totality of polynomials in an indeterminate, $x$, with coefficients in a fixed Galois field of order $p^{n}$. Let $P$ be a primary irreducible element of $\mathfrak{D}$; then, if $A$ is any polynomial in $\mathfrak{D}$ not divisible by $P$,

$$
A^{p^{n \nu}-1} \equiv 1 \quad(\bmod P)
$$

where $\nu$ is the degree of $P$. Evidently then

$$
A^{\left(p^{n \nu}-1\right) /\left(p^{n}-1\right)}
$$

is congruent $(\bmod P)$ to a quantity in the $G F\left(p^{n}\right)$, that is, to a polynomial of degree zero. We define $(A / P)$, the residue character of index $p^{n}-1$, as that element of $G F\left(p^{n}\right)$ for which

$$
\left(\frac{A}{P}\right) \equiv A^{\left(p^{n \nu}-1\right) /\left(p^{n}-1\right)} \quad(\bmod P) .
$$

We have then the following theorem of reciprocity, proved in a recent paper. $\dagger$

If $P$ and $Q$ are primary irreducible polynomials in $\mathfrak{D}$ of degree $\nu$ and $\rho$ respectively, then

$$
\left(\frac{P}{Q}\right)=(-1)^{\rho \nu}\left(\frac{Q}{P}\right) .
$$

The purpose of this note is to give a simple new proof of this theorem along the lines of Zeller's well known proof of the ordinary quadratic reciprocity theorem. $\ddagger$

2. Analog of Gauss' Lemma. If $A$ is in $\mathfrak{D}$, then $\operatorname{sgn} A$ denotes the coefficient of the highest power of $x$ which occurs in $A$; if sgn $A=1, A$ is primary. Let $R(A / B)$ denote the remainder in the division of $A$ by $B$. Then the analog in question is furnished by the following theorem.

* Presented to the Society, December 27, 1932.

$\dagger$ American Journal of Mathematics, vol. 54 (1932), pp. 39-50.

$\ddagger$ Monatsbericht der Berliner Akademie, December, 1872. 
Lemma. Let $A$ and $P$ be in $\mathfrak{D}, P$ primary irreducible, and not a divisor of $A$; then

$$
\left(\frac{A}{P}\right)=\prod_{H} \operatorname{sgn} R\left(\frac{H A}{P}\right),
$$

the product extending over all primary $H$ of degree less than the degree of $P$.

A detailed proof of this lemma is scarcely necessary, but we remark for a later purpose that the proof depends on the fact that the set of polynomials

$$
\left\{R\left(\frac{H A}{P}\right) / \operatorname{sgn} R\left(\frac{H A}{P}\right)\right\}
$$

is identical (except for order) with the set $\{H\}$, where $H$ runs through the primary polynomials of degree less than the degree of $P$.

3. Proof of the Theorem. Let $\{b M\}$ denote the set of those polynomials in the set

$$
\left\{R\left(\frac{H Q}{P}\right)\right\}, \quad(\operatorname{deg} H<\nu, \operatorname{sgn} H=1),
$$

with signum equal to $b$, a fixed quantity in $G F\left(p^{n}\right)$. We write $S_{b}=\{M\}$; evidently the polynomials $M$ are primary. Similarly we put $S_{b}^{\prime}=\{N\}$, where $\{b N\}$ denotes the set of those polynomials in the set

$$
\left\{R\left(\frac{K P}{Q}\right)\right\}, \quad(\operatorname{deg} K<\rho, \operatorname{sgn} K=1),
$$

with signum equal to $b$.

We assume, as we may without any loss in generality, that $\rho \geqq \nu$. We put

$$
S_{b}^{\prime}=U_{b}+V_{b},
$$

where

$$
\begin{aligned}
& U_{b}=\left\{M \text { in } S_{b}{ }^{\prime} ; \operatorname{deg} M<\nu\right\}, \\
& V_{b}=\left\{M \text { in } S_{b}{ }^{\prime} ; \operatorname{deg} M \geqq \nu\right\} .
\end{aligned}
$$


Then we begin by proving

$$
U_{b}=S_{-b}
$$

Indeed, let $M$ be any polynomial in $S_{b}$, that is, let

$$
H Q \equiv b M \quad(\bmod P)
$$

where $\operatorname{deg} H<\nu, \operatorname{sgn} H=1$. Evidently there exists a primary $K$ of degree $<\rho$ such that

$$
H Q=b M+K P .
$$

But this equality may be written in the form

$$
K P \equiv-b M \quad(\bmod Q),
$$

and since $\operatorname{deg} M<\nu$, it follows at once that $M$ is in $U_{-b}$.

Conversely assume an $M$ in $U_{b}$. Then there exists a primary $K$ of degree $<\rho$, such that

$$
K P \equiv b M \quad(\bmod Q)
$$

since deg $M<\nu$, we infer the existence of a primary (in particular, non-zero) $H$ of degree $<\nu$, such that

$$
K P=b M+H Q \text {. }
$$

Then it follows as above that $M$ is in $S_{-b}$. We have therefore set up a $(1,1)$ correspondence between the elements of $U_{b}$ and $S_{-b}$, thus proving equation (4).

Let us write $\mu(W)$ for the number of elements in a (finite) set $W$. Then, by Gauss' Lemma and equation (3),

$$
\left(\frac{P}{Q}\right)=\prod_{b \neq 0} b^{\mu\left(U_{b}\right)+\mu\left(V_{b}\right)},
$$

the product in the right member extending over all $b$ in $G F\left(p^{n}\right)$ different from zero. By equation (4), the right side of (5) may be written in the form

$$
\prod_{b} b^{\mu\left(S_{-b}\right)+\mu\left(V_{b}\right)}
$$

Now

(6) $\prod_{b} b^{\mu\left(S_{-b}\right)}=\prod_{b}(-b)^{\mu\left(S_{b}\right)}=\prod_{b}(-1)^{\mu\left(S_{b}\right)} \cdot \prod_{b} b^{\mu\left(S^{b}\right)}$; 
but (by the remark in $\S 2$ )

$$
\sum_{b} \mu\left(S_{b}\right)=\frac{p^{n \nu}-1}{p^{n}-1} \equiv \nu \quad(\bmod 2),
$$

and by Gauss' Lemma

$$
\prod_{b} b^{\mu\left(S_{b}\right)}=\left(\frac{Q}{P}\right)
$$

we have therefore, by equations $(5), \cdots,(8)$,

$$
\left(\frac{P}{Q}\right)=(-1)^{\nu}\left(\frac{Q}{P}\right) \prod_{b} b^{\mu\left(V_{b}\right)} .
$$

It remains to calculate $\mu\left(V_{b}\right)$; evidently we may ignore the case $b=1$.

Let $M$ be in $V_{b}$, so that for some primary $K$ of degree $<\rho$,

$$
K P \equiv b M \quad(\bmod Q) \text {. }
$$

Since the degree of $M$ is not less than the degree of $P$, we may put

$$
M=A P+c B, \quad \operatorname{deg} B<\nu,
$$

where $A$ and $B$ are primary, and $c$ is in $G F\left(p^{n}\right)$. Then (10) becomes

$$
(K-b A) P \equiv c B \quad(\bmod Q)
$$

But

$$
\operatorname{deg} A=\operatorname{deg} M-\operatorname{deg} P<\rho-\nu,
$$

and since we are assuming $b \neq 1$, we have necessarily

$$
\operatorname{deg} K \geqq \rho-\nu \text {. }
$$

Therefore $K-b A$ is primary and

$$
\rho-\nu \leqq \operatorname{deg}(K-b A)<\rho,
$$

and finally $B$ is in $U_{c}$.

Conversely, let us begin with a $B$ in $U_{c}$ :

$$
K P \equiv c B \quad(\bmod Q), \operatorname{deg} B<\nu .
$$

Then, if $m$ is an integer such that 


$$
\nu \leqq m<\rho,
$$

and $A$ is a primary polynomial of degree $m-\nu$, we have

$$
(K+b A) P \equiv b A P+c B \quad(\bmod Q) ;
$$

and if we put

$$
b A P+c B=b M,
$$

it is evident that $M$ is in $V_{b}$. Indeed

$$
\begin{aligned}
\operatorname{deg}(K+b A) & =\operatorname{deg} K, \\
\operatorname{sgn}(K+b A) & =\operatorname{sgn} K=1 ; \\
\operatorname{deg} M & =\operatorname{deg} A P=m, \\
\operatorname{sgn} M & =b^{-1} \operatorname{sgn}(b A P+c B)=1 .
\end{aligned}
$$

To sum up, we have proved that, for fixed $b \neq 1$,

(i) to each element of $V_{b}$ corresponds a single element of some $U_{c}$;

(ii) to each element of $U_{c}, c$ fixed, corresponds $p^{n(m-\nu)}$ elements of $V_{b}$ of degree $m$, where $m$ is a fixed integer satisfying the inequalities (13).

Evidently (ii) implies that the total number of elements of $V_{b}$ corresponding to a fixed element of $U_{c}$ is precisely

$$
\sum_{m=\nu}^{\rho-1} p^{n(m-\nu)}=\frac{p^{n(\rho-\nu)}-1}{p^{n}-1} .
$$

We have therefore that the number of elements in $V_{b}$ is

$$
\mu\left(V_{b}\right)=\frac{p^{n(p-\nu)}-1}{p^{n}-1} \frac{p^{n \nu}-1}{p^{n}-1},
$$

as follows at once from equations (4) and (7).

Returning to equation (9), we have, since the right member of (14) is independent of $b$,

$$
\prod_{b} b^{\mu\left(V_{b}\right)}=\left(\prod_{b} b\right)^{\epsilon}
$$

$\epsilon$ denoting the right side of (14).

Now, by the generalization of Wilson's Theorem for a Galois field,

$$
\prod_{b} b=-1
$$


on the other hand

$$
\epsilon \equiv(\rho-\nu) \nu \equiv \rho \nu+\nu \quad(\bmod 2) ;
$$

therefore, by (9) and (15),

$$
\left(\frac{P}{Q}\right)=(-1)^{\rho \nu}\left(\frac{Q}{P}\right) \text {. }
$$

This completes the proof of our theorem of higher reciprocity.

4. Remarks. It should be clear that the case $p=2$ is by no means ruled out in the proof just given. Since in the $G F\left(2^{n}\right)$, +1 and -1 are the same, the theorem in this case assumes the simpler form

$$
\left(\frac{P}{Q}\right)=\left(\frac{Q}{P}\right), \quad(p=2),
$$

$(P / Q)$ being the residue character of index $2^{n}-1$.

Secondly, if in the notation of $\$ 3$, we put

$$
W_{b}=\left\{M \text { in } S_{b}^{\prime} ; \operatorname{deg} M \leqq \nu\right\}
$$

and

$$
X_{b}=\left\{M \text { in } S_{b}^{\prime} ; \operatorname{deg} M>\nu\right\},
$$

then it is easy to show that

$$
\prod_{b} b^{\mu\left(X_{b}\right)}=(-1)^{\rho \nu},
$$

or, what amounts to the same thing,

$$
\prod_{b} b^{\mu\left(W_{b}\right)}=\left(\frac{Q}{P}\right) .
$$

Duke University 